\title{
FACTORES DE RIESGO ASOCIADOS AL DESARROLLO DE PIELONEFRITIS AGUDA ENTRE LAS GESTANTES AFILIADAS A UNA EMPRESA ADMINISTRADORA DE PLANES DE BENEFICIO EN CUATRO CIUDADES EN COLOMBIA. ESTUDIO DE CASOS Y CONTROLES
}

\section{Risk factors associated with acute pyelonephritis in pregnant women, from third-party payer institutions in Colombia, between 2008 and 2012. A case-controll study}

Jessika Lissethe López-Martínez, MB, MSc'; Yanett Marcela Montoya-Jaramillo, Candidata $\mathrm{MSc}^{2}$; Dedsy Yajaira Berbesi-Fernández, Enf, $\mathrm{MSc}^{3}$

Recibido: Febrero 28/14 - Aceptado: Diciembre 15/14

\section{RESUMEN}

Objetivo: identificar factores de riesgo relacionados con pielonefritis aguda durante la gestación en las gestantes de Barranquilla, Bogotá, Bucaramanga, Cali y Medellín (Colombia), afiliadas a una empresa administradora de planes de beneficio.

Materiales y métodos: estudio de casos y controles, con dos controles por caso. Criterios de selección: gestantes que presentaron parto entre el 1 de enero de 2008 y 31 de diciembre de 2012, en las diferentes instituciones prestadoras de servicios de salud de una empresa administradora de planes de beneficio en Colombia; se excluyeron aquellas con

1 Microbióloga y bioanalista; magíster en Epidemiología. Epidemiología y Bioestadística, Universidad CES, Medellín (Colombia).

lopezmartinezjesica@gmail.com

2 GESIS, Candidata MSc. Analista de Riesgos, EPS Sura, Medellín (Colombia).

3 Enfermera; magíster en Epidemiología. Docente, Facultad de Medicina, Universidad CES, Medellín (Colombia). anomalías del tracto urinario. Se definieron como caso aquellas que tenían diagnóstico de pielonefritis aguda durante el embarazo, y como control las gestantes con parto atendido en el mismo periodo, sin diagnóstico de pielonefritis. Los casos fueron seleccionados por conveniencia de manera consecutiva, los controles de manera aleatoria. Se midieron las variables gestacionales, sociodemográficas y las comorbilidades. Se evaluó la asociación con análisis bivariado y multivariado.

Resultados: se halló una incidencia de 18 casos de pielonefritis por 10.000 gestantes. El diagnóstico se realizó entre la semana 5 y 38 de gestación, la mayoría durante el segundo (40,2 \%) y tercer (48,0\%) trimestre. Ningún caso presentó antecedentes de pielonefritis. Se halló asociación entre pielonefritis aguda y primigestante (OR = 1,94; IC $95 \%$ : 1,133,33), adolescente (OR = 2,85; IC $95 \%: 1,51-5,40)$ e infección del tracto urinario previa al embarazo $(\mathrm{OR}=2,29$; IC $95 \%: 1,17-4,44)$. 
Conclusión: ser primigestante, adolescente y con antecedente de infección baja del tracto urinario previa al embarazo son factores de riesgo para pielonefritis aguda gestacional.

Palabras clave: pielonefritis, factores de riesgo, embarazo, estudios de casos y controles, Colombia.

\section{ABSTRACT}

Objective: To identify risk factors for the development of acute pyelonephritis in pregnant women in Barranquilla, Bogotá, Bucaramanga, Cali and Medellín (Colombia) covered by a health benefits management organization.

Materials and methods: Case-control study with two controls for every case. Selection criteria: pregnant women presenting for delivery between January 1st, 2008 and December 31st, 2012 to the different healthcare institutions of a thrird-party payer. Women with urinary tract abnormalities were excluded. Cases were defined as those diagnosed with acute pyelonephritis during pregnancy. Controls were defined as pregnant women delivered during the same period, not diagnosed with pyelonephritis. Cases were selected consecutively on the basis of convenience, and controls were selected at random. Gestational, social and demographic variables, and comorbidities were measured. Association was assessed using bivariate and multivariate analysis.

Results: An incidence of 3.5 cases of acute pyelonephritis for every 10,000 pregnant women was found. The diagnosis was made between weeks 5 and 38 of gestation, the vast majority during the second (40.2\%) and the third (48.0\%) trimesters. None of the cases had a history of pyelonephritis. An association was found between acute pyelonephritis, first pregnancy $(\mathrm{OR}=1.94$; IC 95\%: 1.13-3.33), being a teenager $(\mathrm{OR}=2.85$; IC 95\%: 1.51-5.40), and urinary tract infection before pregnancy (OR = 2.29; IC 95\%: 1.17-4.44).

Conclusion: Risk factors for acute gestational pyelonephritis include first pregnancy, being a teenager and having a history of urinary tract infection before pregnancy.
Key words: Pyelonephritis, risk factors, pregnancy, case-control studies, Colombia.

\section{INTRODUCCIÓN}

La pielonefritis aguda o infección del tracto urinario alto se presenta con mayor frecuencia durante la gestación, debido a los cambios inmunológicos, fisiológicos y anatómicos propios de esta etapa $(1,2)$. Su incidencia oscila alrededor del 0,07 al 3,6\% de todas las gestaciones $(3,4)$, y su presencia se asocia con un incremento en la frecuencia de resultados maternos y perinatales adversos. En la madre, la pielonefritis incrementa el riesgo de presentar anemia, bacteremia, síndrome de dificultad respiratoria del adulto, y hospitalización (5); asimismo, aumenta el riesgo de feto pequeño para la edad gestacional, de desprendimiento prematuro de placenta normoinserta y de parto pretérmino (4). El diagnóstico de la pielonefritis aguda se realiza ante el hallazgo de los signos y síntomas sugestivos de la entidad (fiebre, escalofrío, dolor abdominal, náuseas, vómito, disuria, aumento en la frecuencia y urgencia urinaria) $(6,7)$, acompañados de la confirmación objetiva, mediante el urocultivo, de un microorganismo patógeno, siendo en la mayoría de los casos (70\%) la Escherichia coli la implicada $(3,8,9)$. En lo que respecta a su tratamiento, luego de conocerse el resultado del antibiograma, el antibiótico debe ajustarse a las recomendaciones del mismo, para evitar posibles fallas terapéuticas por resistencia bacteriana (10).

En cuanto a los factores de riesgo se ha descrito que la pielonefritis aguda es más frecuente en mujeres nulíparas, con pocos años de educación, más jóvenes, fumadoras y que asisten tarde al control prenatal (5). En Colombia son pocos los estudios publicados respecto a los factores de riesgo relacionados con esta entidad (11).

El objetivo del presente estudio es identificar los factores de riesgo relacionados con la presencia de pielonefritis aguda durante la gestación en las pacientes que consultaron a una empresa administradora de planes de beneficio localizada en las 
ciudades de Barranquilla, Bogotá, Bucaramanga, Cali y Medellín (Colombia), y que recibieron atención médica entre el 1 de enero de 2008 y el 31 de diciembre de 2012.

\section{MATERIALES Y MÉTODOS}

Se realizó un estudio de casos y controles. Se incluyeron las gestantes afiliadas al aseguramiento contributivo en el sistema de seguridad social en Colombia, Plan Obligatorio de Salud (POS), de una empresa administradora de planes de beneficio (EAPB) privada, que opera en las ciudades de Barranquilla, Bogotá, Bucaramanga, Cali y Medellín (Colombia), cuyo control prenatal y partos fueron atendidos entre el 1 de enero de 2008 y el 31 de diciembre de 2012, en la red prestadora de servicios de salud de primer y segundo nivel de esta entidad que atiende a una población de ingresos económicos bajos y medios. Se excluyeron pacientes con diagnóstico de anomalías en el sistema urinario.

Los casos correspondieron a las gestantes con diagnóstico de pielonefritis aguda en la base de datos de la institución, los controles fueron mujeres gestantes que no tuvieron el diagnóstico de pielonefritis aguda (12). En el programa Epidat ${ }^{\circledR}$ versión 3.1 se calculó un tamaño de muestra con una razón de dos controles por cada caso, un poder del $80 \%$ y un nivel de confianza del $95 \%$, con una proporción de casos expuestos (primigestante) del 46,1\% (4) y un OR de 2 para un total de 105 casos y 210 controles. Se seleccionó ser primigestante como la principal variable de asociación porque en la literatura es la variable más frecuentemente reportada. Se incluyeron todos los casos registrados en el periodo de estudio que cumplieron con los criterios de inclusión; los controles se seleccionaron aleatoriamente de la población de referencia, a través de un muestreo aleatorio simple. Los casos y los controles se seleccionaron de un documento de la EAPB denominado informe epidemiológico. Los datos de las variables en estudio se recolectaron de la historia clínica.

Se evaluaron los factores sociodemográficos (edad, adolescente, nivel de ingresos económicos, estado civil), gestacionales (edad gestacional al diagnóstico, primigestante) y comorbilidades (diabetes mellitus gestacional, preeclampsia, vaginitis y antecedente de infección baja del tracto urinario hasta un año antes del embarazo).

Se llevó a cabo un análisis descriptivo de cada una de las variables; a las variables cualitativas se les calcularon frecuencias absolutas y relativas, y a las cuantitativas se les calcularon la mediana (Me) y el rango intercuartílico (RI); previamente se realizó una prueba de normalidad de ShapiroWilk. Se exploró asociación a través de la prueba chi-cuadrado y se calculó Odds Ratio (OR) crudo con su intervalo de confianza (IC) del $95 \%$. Se compararon las medianas a través de la prueba U de Mann-Whitney. Se asumió significancia estadística con un valor $\mathrm{p}<0,05$. Las variables con asociación significativa o aquellas que cumplieron con el criterio de Hosmer-Lemeshow (valor $\mathrm{p}<0,25$ ) se ingresaron a un modelo de regresión logística binaria explicativo a través del método Enter; se muestran OR ajustados con sus respectivos IC al 95\%. Los análisis se realizaron en SPSS® versión 20.0 (Inc., Chicago, Illinoins, USA), licencia amparada por la Universidad CES.

Aspectos éticos. Esta investigación fue avalada por el Comité Institucional de Ética de Investigación en Humanos de la Universidad CES, como consta en el acta de la sesión número 58 de 2013, y por el Comité de Investigación de la EAPB.

\section{RESULTADOS}

En el periodo de estudio se atendieron 62.083 partos y se diagnosticaron 110 casos de pielonefritis aguda, lo que representa una incidencia de 0,18\% de esta infección. Se identificaron 102 casos que cumplieron con los criterios de selección.

Los casos fueron atendidos en Barranquilla $(\mathrm{n}=$ 8), Bogotá $(n=10)$, Bucaramanga $(n=1)$, Cali $(n$ =4) y Medellín ( $n=79)$, y el diagnóstico se realizó entre las semanas 5 y 38 de gestación (mediana $=$ 25,0; Q1 = 18,8; Q3 = 31,0), siendo en la mayoría de los casos durante el segundo (40,2\%) y tercer 
$(48,0 \%)$ trimestre. Ninguno de los casos presentó antecedentes de pielonefritis, pero el $45,1 \%$ de los casos tuvieron antecedente de infección urinaria baja previa al embarazo actual. La tabla 1 muestra la frecuencia de los factores gestacionales, sociodemográficos y comorbilidades en los casos y los controles.

En el análisis univariado se halló asociación entre pielonefritis aguda y ser primigestante $(\mathrm{OR}=2,21$; IC $95 \%$ : 1,32-3,70), ser adolescente (OR = 3,13; IC $95 \%: 1,70-5,76)$, y haber tenido un infección del tracto urinario previa al embarazo actual $(\mathrm{OR}=$ 1,97; IC 95 \%: 1,05-3,70). No se encontró asociación entre pielonefritis y edad gestacional (valor $\mathrm{p}=$ 0,064), nivel de ingresos económicos $(\mathrm{OR}=0,52$; IC $95 \%$ : 0,41-1,67), estado civil soltera $(\mathrm{OR}=1,92$; IC $95 \%$ : 0,89-4,11), diabetes mellitus gestacional $(\mathrm{OR}=0,85$; IC $95 \%$ : 0,22-3,37), preeclampsia (OR
$=1,78 ; \mathrm{IC} 95 \%: 0,77-4,12)$ y vaginitis $(\mathrm{OR}=1,19$; IC 95 \%: 0,67-2,13). El análisis multivariado mostró asociación con ser primigestante, adolescente y el antecedente de infección del tracto urinario (tabla 2).

\section{DISCUSIÓN}

Este estudio estableció una incidencia de pielonefritis aguda de 1,8 x 1000 nacimientos; además, ser primigestante, adolescente y tener antecedente de infección del tracto urinario previo a la gestación actual son factores de riesgo asociados al desarrollo de pielonefritis aguda durante la gestación. Por otra parte, no se halló asociación entre la pielonefritis y el estado civil, así como tampoco con el nivel de ingresos económicos.

La incidencia es más baja que la reportada por Calderón et al., con un 3,6 por 1000 nacidos vivos

\section{Tabla 1.}

Caracterización sociodemográfica y clínica de la población de gestantes con pielonefritis

aguda y sus controles, atendidas en una EAPB del régimen contributivo de Colombia, 2008-2012

\begin{tabular}{|c|c|c|c|}
\hline Variable & $\begin{array}{c}\text { Casos }(n=102) \\
n(\%)\end{array}$ & $\begin{array}{c}\text { Controles }(\mathrm{n}=204) \\
\mathrm{n}(\%)\end{array}$ & Valor $\mathrm{p}$ \\
\hline Primigestante & $74(72,55)$ & $111(54,41)$ & $0,002^{*}$ \\
\hline Edad gestacional (Me \pm RI) & $38 \pm 2$ & $39 \pm 2$ & $0,064^{* *}$ \\
\hline $\operatorname{Edad}(M e \pm R I)$ & $23 \pm 9$ & $25 \pm 7$ & $0,000^{* *}$ \\
\hline Adolescente & $29(28,43)$ & $23(11,27)$ & $0,000^{*}$ \\
\hline Nivel de ingresos económicos & & & $0,379^{*}$ \\
\hline$<2 \mathrm{SM}$ & $94(92,16)$ & $179(87,74)$ & \\
\hline $2-5 \mathrm{SM}$ & $8(7,84)$ & $23(11,27)$ & \\
\hline$>5 \mathrm{SM}$ & $0(0)$ & $2(0,98)$ & \\
\hline Estado civil & & & $0,392^{*}$ \\
\hline Soltera & $50(49,02)$ & $83(40,69)$ & \\
\hline Casada & $11(10,78)$ & $35(17,16)$ & \\
\hline Unión estable & $34(33,33)$ & $72(35,29)$ & \\
\hline Otra & $7(6,86)$ & $14(6,86)$ & \\
\hline Diabetes mellitus gestacional & $3(2,94)$ & $7(3,43)$ & $0,559^{*}$ \\
\hline Preeclampsia & $11(10,78)$ & $13(6,37)$ & $0,176^{*}$ \\
\hline Vaginitis & $23(22,55)$ & $40(19,61)$ & $0,549^{*}$ \\
\hline ITU previa al embarazo actual & $22(21,57)$ & $25(12,25)$ & $0,033^{*}$ \\
\hline
\end{tabular}

SM: salario mínimo; ITU: infección del tracto urinario; Me: mediana; RI: rango intercuartil; NA: no aplica.

"Prueba Chi-cuadrado; ${ }^{* *}$ Prueba U de Mann-Whitney 


\begin{tabular}{|c|c|c|c|}
\hline Variable & OR ajustada & IC al $95 \%$ & Valor $\mathbf{p}$ \\
\hline Primigestante & 1,943 & $1,132-3,335$ & 0,016 \\
\hline Adolescente & 2,858 & $1,512-5,405$ & 0,001 \\
\hline ITU baja antes del embarazo & 2,290 & $1,179-4,440$ & 0,014 \\
\hline Preeclampsia & 1,815 & $0,756-4,356$ & 0,182 \\
\hline
\end{tabular}

IC: intervalo de confianza; OR: odds ratio; ITU: infección del tracto urinario

para Chile en el 2007 (3); el 5 x 1000 informado por Wing et al., para la población afiliada a Káiser permanente durante 1993 a 2010 (5), y el 21 por 1000 mujeres gestantes informado por Sui et al., para Perú entre 1999 y 2000 (13).

Respecto a los factores de riesgo, nuestros resultados concuerdan con Wing et al. (5) en cuanto a que ser adolescente es un factor de riesgo para el desarrollo de pielonefritis aguda durante la gestación. Asimismo, nuestros resultados respecto a las primigestantes concuerdan con lo informado por la literatura $(4,14,15)$. Existe la posibilidad de que haya colinealidad en estas dos variables. Por otra parte, se ha discutido si la adolescencia per se es un factor de riesgo directo, o si la causa es que las gestantes adolescentes pertenecen a estratos socioeconómicos bajos, son fumadoras e incumplen frecuentemente los controles prenatales (16).

Además, se evidenció que la mayoría de los casos de pielonefritis aguda se presentaron en el tercer trimestre; similares resultados han sido reportados en la literatura (5), aunque por otra parte se ha informado que se presentan más en el segundo trimestre (17).

Las fortalezas del estudio son la base poblacional y la selección de los casos y controles de la misma; sin embargo, el diseño de este estudio no permite que las relaciones encontradas sean catalogadas como causales. No obstante, la importancia clínica y la poca evidencia del tema en Colombia hacen que los resultados obtenidos sean relevantes. Pueden haber sesgos de información, a pesar de que se evaluó la calidad de la misma a través de una prueba piloto. Igualmente, es posible que exista sesgo de selección aunque se realizó una selección aleatoria de los controles. Además, la definición de casos podría tener algunas limitaciones por depender de bases de datos hospitalarios.

\section{CONCLUSIÓN}

Ser primigestante, adolescente y con antecedente de infección del tracto urinario bajo en el año previo al embarazo constituyen los factores de riesgo que incrementan la posibilidad de presentar una pielonefritis aguda durante la gestación. Se requieren más estudios que permitan demostrar nuevos factores asociados a esta infección.

\section{AGRADECIMIENTOS}

A la empresa administradora de planes de beneficio EPS SURA por evaluar la investigación a través de su Comité y permitirnos el acceso a la información. $\mathrm{Al}$ doctor José Miguel Abad, coordinador científico nacional de la entidad, por su apoyo permanente. A la Universidad CES por facilitar el uso de sus instalaciones, software y equipos informáticos para la ejecución de esta investigación. Al Comité Institucional de Ética de Investigación en Humanos de la Universidad CES por evaluar esta investigación.

\section{REFERENCIAS}

1. Fiadjoe P, Kannan K, Rane A. Maternal urological problems in pregnancy. Eur J Obstet Gynecol Reprod Biol. 2010;152:13-7. 
2. Foxman B, Klemstine K, Brown P. Acute pyelonephritis in US hospitals in 1997: hospitalization and in-hospital mortality. Ann Epidemiol. 2003;13:144-50.

3. Calderón U, Doren A, Cruz M, Cerda J, Abarzúa C. Pielonefritis aguda en el embarazo y susceptibilidad antimicrobiana de uropatógenos. Comparación de dos décadas. Rev Chil Obstet Ginecol. 2009;74:88-93.

4. Farkash E, Weintraub A, Sergienko R, Wiznitzer A, Zlotnik A, Sheiner E. Acute antepartum pyelonephritis in pregnancy: a critical analysis of risk factors and outcomes. Eur J Obstet Gynecol Reprod Biol. 2012; 162:24-7.

5. Wing DA, Fassett MJ, Getahun D. Acute pyelonephritis in pregnancy: an 18-year retrospective analysis. Am J Obstet Gynecol. 2014;210:219.e1-6. doi: 10.1016/ j.ajog.2013.10.006

6 Gilstrap L, Cunningham F, Whalley P. Acute pyelonephritis in pregnancy: an anterospective study. Obstet Gynecol. 1981;57:409-13.

7. Fahey T, Webb E, Montgomery A, Heyderman R. Clinical management of urinary tract infection in women: a prospective cohort study. Fam Pract. 2003;20:1-6.

8. Pazos N, Fuentes L, Ferrández B, Martínez C, Martínez M, Osuna J. Pyelonephritis and pregnancy. Our experience in a general hospital. An Med Interna Madr Spain. 1984 2007;24:585-7.

9 Czaja C, Scholes D, Hooton T, Stamm W. Populationbased epidemiologic analysis of acute pyelonephritis.
Clin Infect Dis Off Publ Infect Dis Soc Am. 2007; 45:273-80.

10. Pemberthy C, Gutiérrez J, Arango N, Monsalve M, Giraldo N, Gutiérrez F, et al. Aspectos clínicos y farmacoterapéuticos de la infección del tracto urinario. Revisión estructurada. Revista CES Medicina. 2011;25:135-51.

11. Sánchez J, Lomanto A. Factores de riesgo y complicaciones de la infección de vías urinarias durante el embarazo. Rev Colomb Obstet Ginecol. 1991;42: 261-8.

12. Szklo M, Nieto F. Epidemiología intermedia: conceptos y aplicaciones. Madrid: Díaz de Santos; 2003.

13. Sui A, Chung-Joo B. Pielonefritis aguda y gestación. Ginecol Obstet. 2001;47:171-6.

14. Sharma P, Thapa L. Acute pyelonephritis in pregnancy: a retrospective study. Aust N Z J Obstet Gynaecol. 2007;47:313-5.

15. Hill J, Sheffield J, McIntire D, Wendel G. Acute pyelonephritis in pregnancy. Obstet Gynecol. 2005; 105:18-23.

16. Leppälahti S, Gissler M, Mentula M, Heikinheimo O. Is teenage pregnancy an obstetric risk in a welfare society? A population-based study in Finland, from 2006 to 2011. BMJ Open. 2013;3:e003225. doi: 10.1136/bmjopen-2013-003225

17. Sharma $\mathrm{P}$, Thapa L. Acute pyelonephritis in pregnancy: a retrospective study. Aust N Z J Obstet Gynaecol. 2007;47:313-5. 